\title{
Perfil epidemiológico, padrões espaciais e evitabilidade da mortalidade fetal em Pernambuco
}

\author{
Epidemiological profile, spatial patterns and preventability of fetal mortality in Pernambuco \\ Perfil epidemiológico, patrones espaciales y evitabilidad de la mortalidad fetal en Pernambuco
}

\author{
Indianara Maria de Barros Canuto ${ }^{1}$ (B) https://orcid.org/0000-0003-0540-1252 \\ Vilma Costa de Macêdo ${ }^{1}$ ib https://orcid.org/0000-0003-3068-3175 \\ Paulo Germano de Frias ${ }^{3,4}$ id https://orcid.org/0000-0003-4497-8898 \\ Conceição Maria de Oliveira ${ }^{4}$ io https://orcid.org/0000-0002-2220-5782 \\ Cristine Vieira do Bonfim ${ }^{1,5}$ id https://orcid.org/0000-0002-4495-9673
}

Como citar:

Canuto IM, Macêdo VC, Frias PG, Oliveira CM, Bonfim CV. Perfil epidemiológico, padrões espaciais e evitabilidade da mortalidade fetal em Pernambuco. Acta Paul Enferm. 2021;34:eAPE001355.

DOI

http://dx.doi.org/10.37689/actaape/2021A0001355

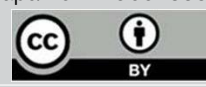

Descritores

Mortalidade fetal; Estatísticas vitais; Análise espacial; Enfermagem em saúde pública; Parto;

Peso ao nascer

Keywords

Fetal mortality; Vital statistics; Spatial analysis; Public health nursing; Parturition; Birth weight

Descriptores

Mortalidad fetal; Análisis espacial; Estadísticas vitales; Enfermería en salud pública; Parto; Peso al nacer

\section{Submetido \\ 15 de Junho de 2020 \\ Aceito \\ 1 de Março de 2021}

Autor correspondente

Indianara Maria de Barros Canuto E-mail: indianaracanuto@hotmail.com

\section{Resumo}

Objetivo: Descrever características epidemiológicas, evitabilidade e distribuição espacial dos óbitos fetais.

Métodos: Estudo ecológico realizado no estado de Pernambuco entre 2010 e 2017, cuja unidade de análise foram regiões de saúde. Utilizou-se dados dos Sistemas de Informações sobre Mortalidade, e sobre Nascidos Vivos. A classificação da evitabilidade dos óbitos seguiu os critérios da Lista brasileira de causas de mortes evitáveis por intervenções do Sistema Único de Saúde. Utilizou-se estatística descritiva e o teste Qui-quadrado para comparações de proporções. Elaborou-se mapas com a distribuição espacial da mortalidade fetal e por causas evitáveis e mal definidas.

Resultados: Registou-se 12.337 óbitos fetais, sendo 8.927 (72,3\%) por causas evitáveis. As variáveis idade da mãe, número de filhos mortos, tipo de gravidez, tipo de parto e peso ao nascer estiveram relacionadas a evitabilidade do óbito. A taxa de mortalidade fetal para o estado de Pernambuco foi de 10,9 por 1000 nascimentos, variando de 10,1 a 16,6, com maior taxa de 16,6 na região XI. A taxa de mortalidade fetal por causas evitáveis foi 7,9, com a mínima de 6,7, e máxima de 13,2 na XI região. A taxa por causas mal definidas foi de 2,3 por 1000 nascimentos, com a maior taxa de 6,2 na IX região.

Conclusão: Os resultados do estudo apresentaram a caracterização dos óbitos fetais, na maior parte evitáveis, e contribuíram para a compreensão da cadeia de fatores envolvidos na ocorrência das mortes. 0 mapeamento das taxas da mortalidade identificou regiões de saúde prioritárias para as ações de redução dos óbitos fetais.

\section{Abstract}

Objective: To describe the epidemiological characteristics, preventability and spatial distribution of fetal deaths.

Methods: Ecological study conducted in the state of Pernambuco between 2010 and 2017 with the health regions as the unit of analysis. Data from Mortality and Live Birth Information Systems were used. The classification of the preventability of deaths followed the criteria of the Brazilian List of causes of preventable deaths by interventions of the National Health Service. Descriptive statistics and the chi-square test were used for comparisons of proportions. Maps with the spatial distribution of fetal mortality and of preventable and ill-defined causes were prepared

Results: There were 12,337 fetal deaths, of which 8,927 (72.3\%) from preventable causes. The variables mother's age, number of dead children, type of pregnancy, type of delivery and birth weight were related to preventability of death. The fetal mortality rate for the state of Pernambuco was 10.9 per 1,000 births, ranging from 10.1 to 16.6 , with a higher rate of 16.6 in region XI. The rate of fetal mortality from preventable causes
'Universidade Federal de Pernambuco, Recife, PE, Brasil.
3Secretaria de Saúde, Recife, PE, Brasil.
${ }^{4}$ Instituto de Medicina Integral Prof. Fernando Figueira, Recife, PE, Brasil.
5Fundação Joaquim Nabuco, Recife, PE, Brasil.
Conflitos de interesse: nada a declarar. 
was 7.9, with a minimum of 6.7 and a maximum of 13.2 in region XI. The rate for ill-defined causes was 2.3 per 1,000 births, and the highest rate was 6.2 in region IX.

Conclusion: The results of the study showed the characterization of fetal deaths, mostly preventable, and contributed to understand the chain of factors involved in the occurrence of deaths. Priority health regions for actions to reduce fetal deaths were identified by mapping the mortality rates.

\section{Resumen}

Objetivo: Describir las características epidemiológicas, la evitabilidad y la distribución espacial de la muerte fetal.

Métodos: Estudio ecológico, realizado en el estado de Pernambuco entre 2010 y 2017, cuyas unidades de análisis fueron regiones de salud. Se utilizaron datos del Sistema de Información sobre Mortalidad y sobre Nacidos Vivos. La clasificación de evitabilidad de las muertes se realizó de acuerdo con los criterios de la Lista brasileña de causas de muertes evitables por intervenciones del Sistema Único de Salud. Se utilizó la estadística descriptiva y la prueba $\chi^{2}$ de Pearson para comparar las proporciones. Se elaboraron mapas con la distribución espacial de la mortalidad fetal por causas evitables y mal definidas.

Resultados: Se registraron 12.337 muertes fetales, de las cuales 8.927 (72,3 \%) fueron por causas evitables. Las variables edad de la madre, número de hijos fallecidos, tipo de embarazo, tipo de parto y peso al nacer estuvieron relacionadas con la evitabilidad de la muerte. El índice de mortalidad fetal en el estado de Pernambuco fue de 10,9 cada 1.000 nacimientos, con una variación de 10,1 a 16,6, y el mayor índice de 16,6 fue en la región Xl. El índice de mortalidad fetal por causas evitables fue de 7,9, con una mínima de 6,7 y una máxima de 13,2 en la región XI. El índice por causas mal definidas fue de 2,30 cada 1.000 nacimientos, con un índice mayor de 6,2 en la región IX.

Conclusión: Los resultados del estudio presentaron la caracterización de las muertes fetales, en su mayoría evitables, y contribuyeron a la comprensión de la cadena de factores relacionados con los casos de muerte. El mapeo de los índices de mortalidad identificó regiones de salud prioritarias para acciones de reducción de muertes fetales.

\section{Introdução}

A mortalidade fetal constitui um problema de saúde pública na maioria dos países. ${ }^{(1)}$ Em particular, por decorrer de condiçôes evitáveis mediante ações de efetivas de saúde e resultar de disparidades socioeconômicas, que caracterizam iniquidades. ${ }^{(2)}$

O óbito fetal ocorre com a morte do produto da gestação antes da expulsão ou da completa extração do organismo materno, independente da duração da gravidez. ${ }^{(3)} \mathrm{O}$ óbito é indicado pela ausência de respiração ou de algum outro sinal de vida após a separação materna. ${ }^{(3)}$

A taxa de mortalidade fetal é um importante indicador de saúde reprodutiva e de qualidade da assistência pré e intra-natal. ${ }^{(4)}$ Segundo a Classificação Estatística Internacional de Doenças e Problemas Relacionados à Saúde $10^{\mathrm{a}}$ Revisão, essa taxa expressa o número de óbitos fetais ocorridos a partir de 22 semanas completas de gestação, ou com peso ao nascer igual ou superior $500 \mathrm{~g}$, comprimento do corpo de $25 \mathrm{~cm}$ ou mais, por mil nascimentos totais na população residente em determinado espaço geográfico, no período considerado. ${ }^{(3)}$

No mundo, ocorrem cerca de 2,6 milhóes de óbitos fetais por ano, aproximadamente metade no momento do parto, e a maioria são mortes evitáveis. ${ }^{(5)} \mathrm{A}$ taxa de mortalidade fetal em 2015 foi de 18,4 por 1000 nascimentos, $25,5 \%$ menor que a taxa do ano
2000 de 24,7 por 1000 nascimentos. ${ }^{(6)}$ No Brasil, nesse mesmo período a taxa decresceu $8,9 \%$, de 12,2 para 10,8 por 1000 . No ano de 2015 , a regiáo Nordeste apresentou a maior taxa do país com 12,1 por 1000 nascimentos. ${ }^{(7)}$

Apesar da magnitude desse indicador, a mortalidade fetal esteve secundarizada nas agendas de políticas nacionais e internacionais. ${ }^{(8)} \mathrm{A}$ discussão sobre os óbitos fetais não foi incluída nos Objetivos do Desenvolvimento do Milênio (ODM), nem nos Objetivos do Desenvolvimento Sustentável (ODS) da Organização das Naçôes Unidas (ONU), acordos mundiais que apresentaram metas para o declínio da mortalidade materna e infantil. ${ }^{(9)}$ Os óbitos fetais têm ganhado maior visibilidade após serem inseridos no Every Newborn Action Plan, movimento global para a eliminação da mortalidade fetal evitável e diminuição de disparidades na sua ocorrência. ${ }^{(8)}$

A invisibilidade histórica dos óbitos fetais nas políticas de saúde materna e infantil dificultaram a redução mais expressiva da mortalidade fetal, especialmente nas áreas de maior vulnerabilidade social. ${ }^{\left({ }^{8}\right)}$ No Brasil e no mundo, a distribuição desigual da mortalidade no território desvela segregaçóes entre grupos populacionais, segundo questôes relacionadas a escolaridade, trabalho, renda, situação do local de moradia e acesso à saúde. ${ }^{(10)}$

A elaboração de pesquisas sobre mortalidade fetal permite ampliar a compreensão sobre os fatores que 
influenciam a ocorrência dessas mortes. ${ }^{(1)}$ Pois, possibilitam a identificaçáo de desigualdades sociais nos grupos populacionais e o monitoramento da ocorrência dos óbitos em diferentes espaços geográficos. ${ }^{(2)}$ Ao verificar a distribuição espacial da mortalidade, esses estudos podem subsidiar o planejamento de açôes, direcionadas a redução dos óbitos fetais evitáveis em populações e territórios vulneráveis. ${ }^{(2,12)}$

Este estudo justifica-se pela magnitude dos óbitos fetais, apesar da maior parte deles ser prevenível pela adequada assistência à saúde pré-natal e obstétrica. Essas mortes ainda são secundarizadas nas pesquisas epidemiológicas, se comparada à mortalidade materna e infantil. Além disso, poucos trabalhos consideram a influência dos determinantes sociais da saúde na dinâmica espacial dos óbitos fetais.

O objetivo deste estudo foi descrever características epidemiológicas, evitabilidade e distribuição espacial dos óbitos fetais.

\section{Métodos}

Foi realizado um estudo ecológico no estado de Pernambuco, que apresenta extensão territorial de $98.076,021 \mathrm{~km}^{2}$ e abriga uma população de 9.496.294 habitantes. ${ }^{(13)} \mathrm{A}$ unidade de análise do estudo foram as 12 regióes de saúde pernambucanas: I (19 municípios), II (20 municípios), III (22 municípios ), IV (32 municípios), V (21 municípios), VI (13 municípios), VII (7 municípios), VIII (7 municípios), IX (11 municípios), X (12 municípios), XI (10 municípios) e XII (10 municípios).

As fontes de dados foram os registros do Sistema de Informações sobre Mortalidade e do Sistema de Informações sobre Nascidos Vivos. Foram incluídos todos os óbitos fetais de mães residentes em Pernambuco, registrados no Sistema de Informaçôes sobre Mortalidade no período de 2010 a 2017.

Para classificação da evitabilidade dos óbitos utilizou-se a Lista brasileira de causas de mortes evitáveis por intervençôes do Sistema Único de Saúde. A listagem categoriza as mortes em: evitáveis (reduzíveis por ações de imunoprevenção, por adequada atenção à mulher na gestação, ao parto e ao recém-nascido; por açóes adequadas de diagnóstico e tratamento; por ações adequadas de promoção e atenção à saúde); causas mal definidas (sintomas, sinais e achados anormais de exames clínicos e laboratoriais não classificados em outra parte; morte fetal de causa não especificada; afecçóes originadas no período perinatal, não especificadas) e demais causas não claramente evitáveis (as demais causas e mortes). ${ }^{(14)}$

Calcularam-se as taxas de mortalidade fetal (número de óbitos fetais dividido pelo total de nascimentos multiplicado por 1000): por causas evitáveis, por causas mal definidas e por causas não claramente evitáveis. Para os óbitos fetais por categoria de evitabilidade analisaram-se variáveis referentes às características maternas: idade da mãe em anos $(<20,20$ 34 e $>34)$, escolaridade da mãe em anos $(<9$ e $\geq 9)$, número de filhos vivos (Nenhum e $\geq 1$ ), número de filhos mortos (Nenhum e $\geq 1$ ); da gestação: tipo de gravidez (única e dupla ou tripla), semanas de gestação (<37 e $\geq 37$ ); do parto: tipo de parto (vaginal e cesáreo); e do nascimento: peso ao nascer $(<2500 \mathrm{~g}$ e $\geq 2500 \mathrm{~g}$ ) e sexo (masculino e feminino). Aplicou-se estatística descritiva e teste Qui-quadrado com nível de significância $<0,05$ para comparação de proporçóes por meio do programa $\mathrm{R}$ versão 3.6 ${ }^{\circ}$.

Verificou-se a distribuição espacial por meio de mapas temáticos da mortalidade fetal e por causas evitáveis e não definidas nas regióes de saúde, elaborado pelo programa QGis ${ }^{\oplus}$ versão 2.14.3. Os dados foram agrupados por tercis e as taxas de mortalidade foram estratificadas em baixa, média e alta. A malha digital usada se encontra disponível no site do Instituto Brasileiro de Geografia e Estatística (IBGE) ${ }^{(15)}$ e apresenta sistema de referências de coordenadas Sirgas 2000/UTM zone 25S.

O projeto de pesquisa foi aprovado pelo Comitê de Ética em Pesquisa do Centro de Ciências da Saúde da Universidade Federal de Pernambuco (CAEE: 13981419.6.0000.5208).

\section{Resultados}

No período do estudo, ocorreram 12.337 óbitos fetais, dos quais $8.927(72,4 \%)$ foram por causas evitáveis. A taxa de mortalidade fetal foi 10,9 por 1000 nascimentos, a taxa por causas evitáveis foi 7,9 e a taxa por causas mal definidas foi de 2,3 (Tabela 1). 
Tabela 1. Número, proporção e taxa dos óbitos fetais segundo os critérios de evitabilidade

\begin{tabular}{lcc}
\hline Evitabilidade $^{*}$ & $\mathbf{n}(\%)$ & $\begin{array}{c}\text { Taxa de } \\
\text { mortalidade }\end{array}$ \\
\hline Causas evitáveis & $8927(72,4)$ & 7,9 \\
Causas de morte mal definidas & $2628(21,3)$ & 2,3 \\
Demais causas (não claramente evitáveis) & $780(6,3)$ & 0,7 \\
Total & $12337(100)$ & 10,9 \\
\hline
\end{tabular}

número/percentual de ignorados: $2 /<0,1 \%{ }^{*}$

Tabela 2. Características dos óbitos fetais segundo os critérios de evitabilidade da Lista brasileira de causas de mortes evitáveis por intervenções do Sistema Único de Saúde

\begin{tabular}{|c|c|c|c|}
\hline \multirow[b]{2}{*}{ Variáveis } & \multicolumn{2}{|r|}{ Óbito fetal } & \multirow[b]{2}{*}{$p$-value } \\
\hline & $\begin{array}{c}\text { Causas } \\
\text { evitáveis } \\
\mathrm{n}(\%)\end{array}$ & $\begin{array}{c}\text { Causas mal } \\
\text { definidas/não } \\
\text { claramente evitáveis } \\
\mathrm{n}(\%)\end{array}$ & \\
\hline \multicolumn{4}{|c|}{ Idade da mãe em anos [n=10.921 (a)] } \\
\hline$<20$ & $1181(70,2)$ & $502(29,8)$ & \multirow{3}{*}{0,009} \\
\hline $20-34$ & $5489(73,8)$ & $1947(26,2)$ & \\
\hline$>34$ & $1322(73,4)$ & $480(26,6)$ & \\
\hline \multicolumn{4}{|c|}{ Escolaridade da mãe em anos [n= 9.948 (b)] } \\
\hline$<9$ & $3949(72,5)$ & $1497(27,5)$ & \multirow{2}{*}{0,238} \\
\hline$\geq 9$ & $3312(73,6)$ & $1190(26,4)$ & \\
\hline \multicolumn{4}{|c|}{ Número de filhos vivos [ $\mathrm{n}=10.586$ (c)] } \\
\hline Nenhum & $3265(72,9)$ & $1213(27,1)$ & \multirow[t]{2}{*}{0,446} \\
\hline$\geq 1$ & $4494(73,6)$ & $1614(26,4)$ & \\
\hline \multicolumn{4}{|c|}{ Número de filhos mortos $[\mathrm{n}=10.256$ (d)] } \\
\hline Nenhum & $2972(70,8)$ & $1223(29,2)$ & \multirow{2}{*}{$<0,001$} \\
\hline$\geq 1$ & $4541(74,9)$ & $1520(25,1)$ & \\
\hline \multicolumn{4}{|c|}{ Tipo de Gravidez [n= $11.545(\mathrm{e})]$} \\
\hline Única & $7976(73,1)$ & $2940(26,9)$ & \multirow{2}{*}{0,021} \\
\hline Dupla ou tripla & $486(77,3)$ & $143(22,7)$ & \\
\hline \multicolumn{4}{|c|}{ Semanas de gestação $[n=10.295(f)]$} \\
\hline$<37$ & $5220(73,8)$ & $1853(26,1)$ & \multirow{2}{*}{0,918} \\
\hline$\geq 37$ & $2381(73,9)$ & $841(26,1)$ & \\
\hline \multicolumn{4}{|c|}{ Tipo de parto $[\mathrm{n}=11.456(\mathrm{~g})]$} \\
\hline Vaginal & $6017(70,0)$ & $2579(30,0)$ & \multirow{2}{*}{$<0,001$} \\
\hline Cesáreo & $2372(82,9)$ & $488(17,1)$ & \\
\hline \multicolumn{4}{|c|}{ Peso ao nascer $[n=11.130(h)]$} \\
\hline$<2500 \mathrm{~g}$ & $5519(72,5)$ & $2097(27,5)$ & \multirow{2}{*}{$<0,001$} \\
\hline$\geq 2500 \mathrm{~g}$ & $2668(75,9)$ & $846(24,1)$ & \\
\hline \multicolumn{4}{|c|}{$\operatorname{Sexo}[n=11.645(i)]$} \\
\hline Masculino & $4500(73,7)$ & $1609(26,3)$ & \multirow[t]{2}{*}{0,084} \\
\hline Feminino & 3999(72,2) & $1537(27,8)$ & \\
\hline
\end{tabular}

Número/ percentual de ignorados (a) 1.416/11,5\%; (b)2.389/19,4\%; (c)1.751/14,2\%; (d)2.081/16,9\%; (e) $792 / 6,4 \%$; (f)2.042/16,6; (g) $881 / 7,1 \%$; (h) $1.202 / 9,8 \%$; (i) $692 / 5,6 \%$

A comparação das características maternas entre os óbitos fetais evitáveis e os não claramente evitáveis mostrou diferença estatisticamente significante entre a idade materna e o número de filhos mortos. A faixa etária maior que 34 anos verificou-se principalmente óbitos evitáveis $1.322(73,4 \%)$, semelhantemente a de 20 a 34 anos 5.489 (73,8\%). Com relação as características da gestação parto e nascimento, entre os óbitos evitáveis predominaram gravidez dupla ou tripla $(n=486 ; 77,3 \%)$, parto cesáreo $(\mathrm{n}=2372 ; 82,9 \%)$ e peso inferior a $2500 \mathrm{~g}$ ( $\mathrm{n}=5519 ; 72,5 \%)$ (Tabela 2).

A mortalidade fetal apresentou maior taxa de 16,6 por 1000 nascimentos na XI região de saúde, e a menor taxa de 10,1 por 1000 na $X$ regiáo. A mortalidade por causas evitáveis variou de 6,7 na $X$ região até 13,2 por 1000 na XI região. A mortalidade por causas mal definidas taxa máxima de 6,2 na IX região (Figura 1).

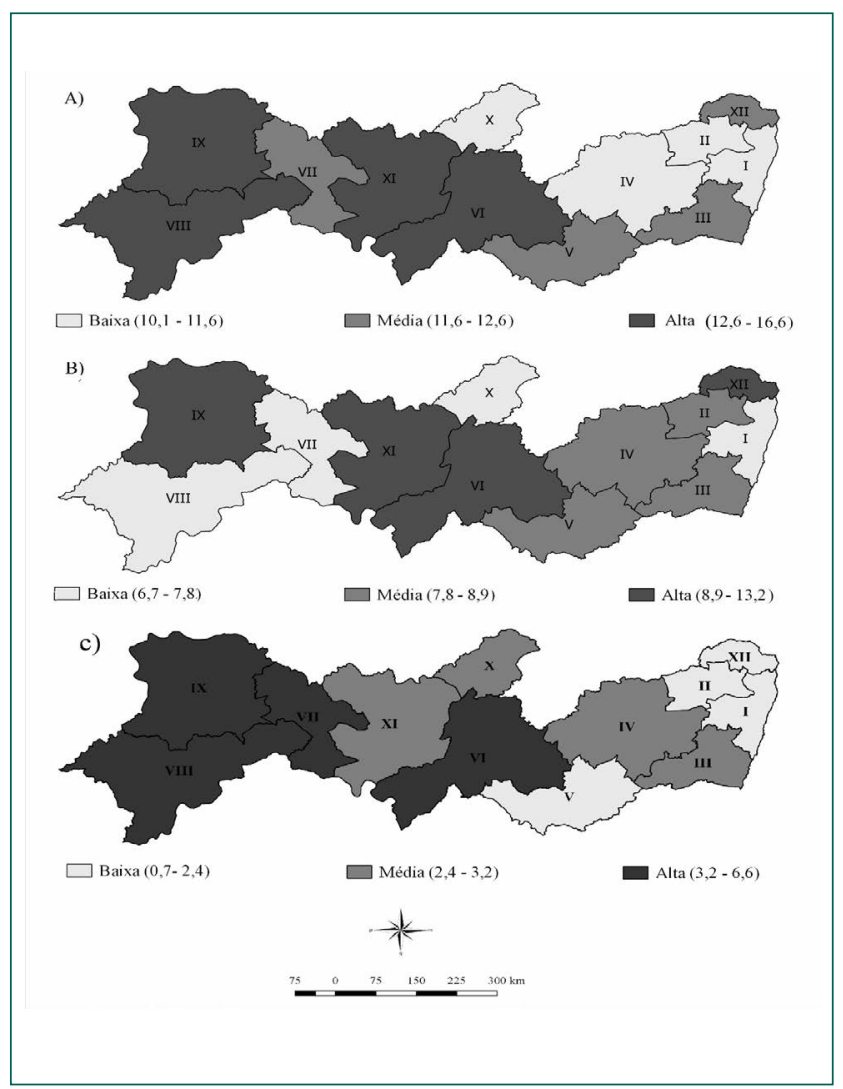

Figura 1. Distribuiç̧ão espacial da mortalidade fetal (A) e por causas evitáveis (B) e por causas não definidas (C), segundo região de saúde

\section{Discussão}

No período estudado, a maior parte dos óbitos fetais ocorreu por causas evitáveis. As características maternas referentes aos extremos de idade e a morte anterior de outros filhos relacionaram-se a evitabilidade dos óbitos fetais. As gestações duplas ou triplas, os nascimentos de parto cesáreo e com baixo peso ocorreram principalmente nos óbitos fetais evitáveis. A XI região de saúde apresentou simul- 
taneamente as maiores taxas de mortalidade fetal e por causas evitáveis. Enquanto a taxa mais elevada de mortalidade por causas náo definidas ocorreu na IX região.

A faixa etária materna superior a 34 anos apresentou principalmente óbitos fetais evitáveis. A literatura demonstra resultados perinatais adversos, risco de morte fetal 50\% maior em gravidez tardia quando comparado a gestantes em outras faixas etárias. ${ }^{(16)}$ Gestaçôes tardias estão mais propícias a patologias específicas, como diabetes e hipertensão gestacionais, com repercussóes negativas ao feto, ${ }^{(17)}$ e apresentam maior incidência de malformações congênitas e deformidades incompatíveis com a vida. ${ }^{(17)}$

A maior parte dos partos cesáreos ocorreram nos fetos com causas de morte evitáveis. É observado em pesquisa que para gestantes com concepto morto decorrente de urgência obstétrica é indicado o parto cesáreo para a preservação da vida materna. ${ }^{(18)}$ Já a prática de cesarianas eletivas é capaz de aumentar o risco ao parto prematuro e ao óbito fetal, sendo mais recomendado a via de parto vaginal quando há boas condiçôes de vitalidade. ${ }^{(19)}$

A mortalidade foi superior entre os fetos com baixo peso ao nascer. Há consenso que quanto menor o peso ao nascer maior o risco a mortalidade fetal e infantil. ${ }^{(20,21)}$ Para reduzir os nascimentos com peso insuficiente é importante o acesso ao pré-natal com início no primeiro trimestre de gravidez, frequência adequada nas consultas e assistência de qualidade. ${ }^{(22)}$

O cuidado pré-natal efetivo é capaz de promover saúde, prevenir, diagnosticar e tratar doenças, com manejo adequado para reduzir os nascimentos com baixo peso e consequentes óbitos fetais evitáveis. ${ }^{(22,23)}$ Pesquisa aponta que as mortes fetais ocorrem principalmente no momento anteparto, por condiçóes maternas que poderiam ter sido prevenidas, identificadas, acompanhadas e controladas com uma assistência pré-natal apropriada. ${ }^{(24)}$ Enquanto os óbitos intrapartos seriam passíveis de evitabilidade, sobretudo pela melhora nas condiçóes de cuidado durante o parto com acesso oportuno a serviços de qualidade. ${ }^{(24)}$

As boas práticas durante o trabalho de parto e parto incluem a assistência humanizada, sem in- tervenções desnecessárias, e conduzida por equipe multidisciplinar, que acolha e acompanhe a gestante desde a sua admissão no serviço. ${ }^{(25)} \mathrm{O}$ acesso a unidades de saúde com recursos estruturais e humanos suficientes para uma assistência obstétrica adequada também são imprescindíveis para a sobrevivência fetal. Ao passo que a peregrinaçáo da gestante em busca do acesso a rede hospitalar aumenta o risco de morte evitável da mãe e do concepto. ${ }^{(26)}$

As causas de morte evitáveis estiveram em maior proporção entre os óbitos fetais e são consideradas eventos sentinelas. $\mathrm{O}$ monitoramento de acontecimentos indesejáveis são capazes de indicar a qualidade da atenção prestada pelos sistemas de saúde, ${ }^{(27)}$ permitindo avaliar o desempenho dos serviços e estabelecer comparaçóes entre regióes e municípios. ${ }^{(28)}$

As maiores taxas de mortalidade fetal e por causas evitáveis foram encontradas na XI região de saúde, e a taxa mais elevada de mortalidade por causas mal definidas na IX região. Municípios dessas regióes estiveram entre os dez mais baixos índices de desenvolvimento humano municipal na dimensão renda de acordo com dados do ano de 2010. ${ }^{(29)}$ Estudos recomendam que nas regióes com altas taxas de mortalidade infantil e fetal, sejam fortalecidas políticas públicas intersetoriais e ações com vistas a ampliação da cobertura da Estratégia de saúde da família e qualificação da assistência ao parto. ${ }^{(2,30)}$

Outra iniciativa que pode auxiliar a redução da mortalidade nessas regiôes é a atuação da vigilância do óbito infantil e fetal. ${ }^{(31)}$ Essa iniciativa também têm contribuído com a retificação das causas básicas, o que permite a correta especificação e o enquadramento adequado na classificação de evitabilidade. ${ }^{(31)}$

A confiabilidade dos sistemas de informaçóes permite a elaboração de estudos como este, utilizando dados secundários. Possibilita também à gestấo pública o conhecimento sobre os grupos populacionais com maior risco de morte, por meio da elaboração de indicadores de saúde pública. ${ }^{(32)} \mathrm{No}$ estado de Pernambuco, as informaçôes vitais são considerados confiáveis, mas a medida que distanciam-se da capital existem municípios com informações em fase de consolidação, ainda que poucos, que apresentam cobertura e qualidade dos registros incompletos. ${ }^{(33)}$ 
A distribuição espacial das taxas de mortalidade fetal realizada nesse estudo contribui com a identificação das regióes de saúde com maiores taxas e podem colaborar na elaboração de estratégias de reduçáo das desigualdades da mortalidade. A análise espacial é capaz de subsidiar os gestores da saúde na definição de áreas que necessitam de prioridade em ações assistenciais e de vigilância em saúde. Pesquisas sobre mortalidade fetal têm usado dados georreferenciados para verificar a distribuição espacial dos riscos ao óbito no território. ${ }^{(2,30)}$ Mas também para monitorar a ocorrência das mortes, auxiliar no desenvolvimento de açóes e programas de saúde pública direcionadas as necessidades da população, além de acompanhar o desempenho de estratégias propostas para a redução da mortalidade. ${ }^{34,35)}$

Este estudo apresenta como limitaçóes a possível subnotificação dos óbitos e incompletude dos sistemas de informação, que pode influenciar no cálculo das taxas, porém a completitude e a confiabilidade dos registros vitais no estado de Pernambuco são considerados adequados. Outra limitação é a utilização da Lista brasileira de causas de mortes evitáveis, que não é exclusiva para óbitos fetais, mas contempla os óbitos neonatais que apresentam circunstâncias e etiologias semelhantes a das mortes fetais. É possível que a unidade de análise região de saúde abrigue desigualdades espaciais, contudo essas unidades são usadas rotineiramente pela gestão da saúde e os resultados desse estudo podem subsidiar o planejamento e a execução de políticas públicas.

\section{Conclusão}

Os resultados do estudo apresentaram a caracterização dos óbitos fetais, na maior parte evitáveis, e contribuíram para a compreensão da cadeia de fatores envolvidos na ocorrência das mortes. As variáveis idade da mãe número de filhos mortos, tipo de gravidez, tipo de parto e peso ao nascer mostraram diferença estatisticamente significante entre os óbitos fetais evitáveis e não claramente evitáveis. As maiores taxas de mortalidade e de mortalidade por causas evitáveis ocorreram respectivamente nas regióes XI e IX. O mapeamento identificou áreas prioritárias para o desenvolvimento de açóes de vigilância em saúde e de melhoria da qualidade da assistência materna e infantil.

\section{Agradecimentos}

O presente trabalho foi realizado com o apoio da Coordenação de Aperfeiçoamento de Pessoal de Nível Superior - Brasil (CAPES) código de financiamento 001.

\section{Colaborações}

Canuto IMB, Bonfim CV e Macêdo VC contribuíram com a concepção do estudo, análise e interpretação dos dados, redação do manuscrito, revisão crítica e de seu conteúdo intelectual. Oliveira CM e Frias PG contribuíram com a análise e interpretação dos dados e redaçáo do manuscrito. Todos os autores aprovaram a versão final do manuscrito.

\section{Referências}

1. Egbe TO, Ewane EN, Tendongfor N. Stillbirth rates and associated risk factors at the Buea and Limbe regional hospitals, Cameroon : a casecontrol study. BMC Pregnancy and Childbirth. 2020;8:1-8.

2. Loiacono, KV. Evolution of fetal mortality in the setting of Argentine socioeconomic inequalities . Period 2007-2014. Arch Argent Pediatr. 2018;116(4):567-74.

3. World Health Organization. International Statistical Classification of Diseases and Related Health Problems: 10. revision. 2.ed. Geneva: WHO; 2004.

4. Dev A, O'Hern K, Domerçant JY, Lucien G, Lafortune L, Grand-Pierre R, et al. A retrospective review of facility-level obstetric complications and stillbirths in southern Haiti, 2013 - 2016. Rev Panam Salud Publica. 2019;(3):1-8.

5. Aminu M, Bar-Zeev S, White S, Mathai M, Broek NVD. Understanding cause of stillbirth : a prospective observational multi-country study from sub-Saharan Africa. BMC Pregnancy and Childbirth. 2019;7:110.

6. Blencowe H, Cousens S, Jassir FB, Say L, Chou D, Mathers C, et al. National , regional , and worldwide estimates of stillbirth rates in 2015 , with trends from 2000 : a systematic analysis. The Lancet Global Health. 2016;4(2):98-108.

7. Brasil, Ministério da saúde. Departamento de informática do SUS, 2018. Brasília (DF): Ministério da Saúde; 2018. [citado 2021 Jan 25]. Disponível em: <http://datasus.saude.gov.br/informacoes-de-saude/ tabnet/estatisticas-vitais>. 
8. Nonterah EA, Agorinya IA, Kanmiki EW, Kagura J, Tamimu M, Ayamba EY et al. Trends and risk factors associated with stillbirths : A case study of the Navrongo War Memorial Hospital in Northern Ghana. Plos one. 2020;1-13.

9. Iqbal S, Maqsood S, Zakar R, Zakar MZ, Fischer F. Continuum of care in maternal, newborn and child health in Pakistan : analysis of trends and determinants from 2006 to 2012. BMC Health Serv Res. 2017;17(189):1-15.

10. Barros P S, Aquino EC, Souza MR. [Fetal mortality and the challenges for women's health care in Brazill. Rev Saude Publica. 2019; 53:(12)110. Portuguese.

11. Smith RB, Beevers SD, Gullivera J, Dajnak D, Fecht D, Blangiardo M, et al. Impacts of air pollution and noise on risk of preterm birth and stillbirth in London. Environment International. 2020;134:1-12.

12. Callander EJ, Hons JTBA, Fox MPH, Ellwood D, Flenady V. What are the costs of stillbirth ? Capturing the direct health care and macroeconomic costs in Australia. Birth, n. September. 2019;00:1-8.

13. Instituto Brasileiro de Geografia e Estatística (IBGE). Portal On-line Cidades. Rio de Janeiro: IBGE; 2018. [citado 2018 Dez 15]. Disponível em: <https:// cidades.ibge.gov.br/brasil/pe/panorama>. Acesso em: 15 dez. 2018.

14. Malta DC, Prado RR, Saltarelli RMF, Monteiro RA, Souza MFM, Almeida MF. [Update of avoidable causes of deaths due to interventions at the Brazilian Health System]. Epidemiol Serv Saúde. 2010;19(2):173-6. Portuguese.

15. Instituto Brasileiro de Geografia e Estatística (IBGE). Malhas digitais. 2020. Rio de Janeiro: IBGE; 2020. [citado 202018 Dez 15]. Disponível em:<https://mapas.ibge.gov.br/en/bases-e-referenciais/basescartograficas/malhas-digitais>.

16. Dongarwar D, Aggarwal A, Barning K, Salihu HM. Stillbirths among Advanced Maternal Age Women in the United States: 20032017. International Journal of Maternal and Child Health and AIDS. 2020;9(1):153-6.

17. Hidalgo-Lopezosa P, Cobo-Cuenca Al, Carmona-Torres JM, Luque-Carrillo P, Rodríguez-Muñoz PM, Rodríguez-Borrego MA. Factors associated with late fetal mortality. Archives of Gynecology and Obstetrics. 2018;296(6):145-1420.

18. Lima KJ, Chaves CS, Gomes EO, Lima MA, Candeira ECP, Teófilo EKS, et al. [Health situation analysis: fetal mortality in the health region of Ceará]. Revista Brasileira em Promoção da Saúde. 2017;30(1):30-37. Portuguese.

19. Mascarello KC, Horta BL, Silveira MF. [Maternal complications and cesarean section without indication: systematic review and metaanalysis]. Rev Saude Publica. 2017;51:105. Portuguese.

21. Garcia LP, Fernandes CM, Traebert J.[ Risk factors for neonatal death in the capital city with the lowest infant mortality rate in Brazil]. Jornal de Pediatria. 2019;95(2):194-200. Portuguese.

22. Esteves APP, Viellas EF, Domingues RMSM, Gama SGN. [Prenatal care in the Brazilian public health services]. Rev. SaúdePública, 2020;54:08. Portuguese.
23. Monteiro MFV, Barbosa CP, Vertamatti MAF, Tavares MNA, Carvalho ACO, Alencar APA. [Access to public health services and integral care for women during the puerperal gravid period period in Ceará, Brazil. BMC Health Services Research]. 2019;19(851):1-8. Portuguese.

24. Salazar-Barrientos M, Zuleta-Tobón JJ. Application of the International Classification of Diseases for Perinatal Mortality (ICDPM) to vital statistics records for the purpose of classifying perinatal deaths in Antioquia, Colombia. Rev Colomb Obstet Ginecol. 2019; 70:228- 242.

25. Leal MC, Bittencourt SA, Esteves PAP, Ayres BVS, Silva LBRAA, Thomaz EBAF, et al. [Avances en la asistencia al parto en Brasil: resultados preliminares de dos estudios evaluativos]. Cad. Saúde Pública [Internet]. 2019; 35(7): e00223018. Portuguese.

26. Lima SS, Braga MC, Vanderlei LCM, Luna CF, Frias. [Assessment of the impact of prenatal, childbirth, and neonatal care on avoidable neonatal deaths in Pernambuco State, Brazil: an adequacy study]. Cadernos de Saude Publica. 2020;36(2). Portuguese.

27. Malta DC, Prado RR, Saltarelli RMF, Monteiro RA, Souza MFM, Almeida MF. [Preventable deaths in childhood, according to actions of the Unified Health System, Brazil]. Rev Bras Epidemiol. 2019;22:1-15. Portuguese.

28. Berman Y, Ibiebele I, Patterson JA, Randal D, Ford JB, Nippita T, et al. Rates of stillbirth by maternal region of birth and gestational age in New South Wales, Australia 2004 - 2015. Aust N Z J Obstet Gynaecol. 2019:1-8

29. Atlas do desenvolvimento humano no Brasil. Rio de Janeiro, PNUD, IPEA, Fundação João Pinheiro, 2020.

30. Canuto IMB, Alves FAP, Oliveira CM, Frias PG, Macêdo VC, Bonfim CV. [Intraurban differentials of perinatal mortality: modeling for identifying priority areas]. Rev Esc Anna Nery. 2019; 23(1):1-8. Portuguese.

31. Oliveira CM, Bonfim CV, Guimarães MJ, Frias PG, Medeiros ZM. [Infant mortality: temporal trend and contribution of death surveillance]. Acta Paul Enferm. 2016;29(3):282-90. Portuguese

32. Figueirôa $B Q$, Frias $P G$, Vanderlei LC, Vidal SA, Carvalho PI, Pereira $\mathrm{CC}$, et al. [Evaluation of the implantation of the Mortality Information System in Pernambuco state, Brazil, in 2012]. Epidemiol. Serv. Saúde. 2019;28( 1 ): e2018384. Portuguese.

33. Szwarcwald CL, Leal MC, Esteves- Pereira AP, Almeida WS, Frias $P G$, Damacena GN, et al. [Evaluation of data from the Brazilian Information System on Live Births (SINASC)]. Cad. Saúde Pública 2019;35(10):e00214918. Portuguese.

34. Adeyinka DA, Olakunde B0, Muhajarine N. Evidence of health inequity in child survival : spatial and Bayesian network analyses of stillbirth rates in 194 countries. Scientific Reports. 2019;9:1-11.

35. Luque-Fernandez MA, Thomas A, Gelaye B, Racape J, Sanchez MJ, Williams MA. Secular trends in stillbirth by maternal socioeconomic status in Spain 2007 - 15: a population-based study of 4 million births. European Journal of Public Health. 2019;29(6):1043-8. 